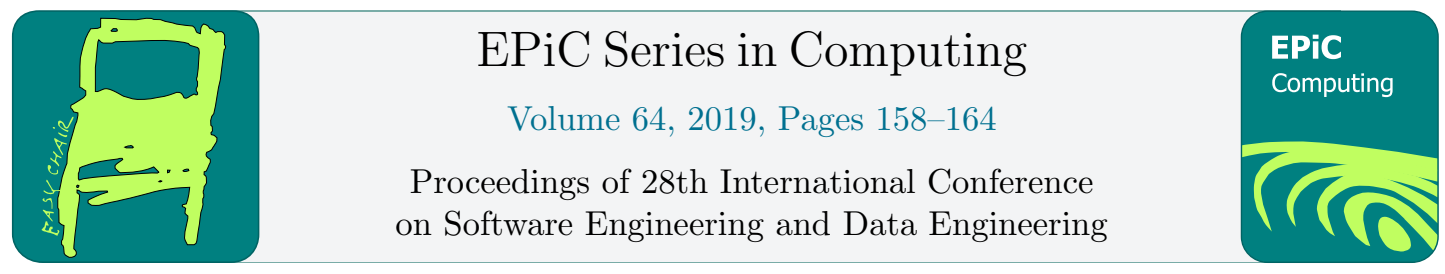

\title{
Cloud Based Framework to Integrate Map, GPS, and Android Apps
}

\author{
Milad A. Khalil and Jiang Guo * \\ California State University, Los Angeles, USA \\ milad.khalil@gmail.com, jguo@calstatela.edu
}

\begin{abstract}
Our cloud based Smart Order Online System is a cloud based and context-aware online retailing system with real time updates. It is designed to verify our framework and handle the operations of various types of e-commerce business. It is aimed at helping customers to obtain order information through cloud based platforms such as mobile devices, laptops or desktop computers. The system allows customers to place orders, to find location and direction to branches, and allows managers to track the delivering drivers' locations and display them on the map. The clustering/grouping algorithm plays a very important role in our framework. It is also discussed in the paper.
\end{abstract}

\section{Introduction}

Cloud and Internet technologies have made a dramatic change on commerce operations, such as online orders [1]. The Cloud Based Smart Order Online System (SOOS) is designed to offer users a better way to search, place, track and manage orders.

The SOOS provides the user with the ability to place orders, group orders, track drivers and orders on map, make adjustments. It allows administrators to make changes to product descriptions, product pricing, product categorizing, and other details regarding various products that are displayed online. This complete control over inventory is reflected immediately to the storefront. Also, SOOS gives administrators the necessary tools to manage customers efficiently and effectively. Administrator can access customer information such as username, contact info, shipping profiles, and credit card and other billing information all found in a single, organized location.

More important, the SOOS provides a clustering/grouping algorithm to group orders based on their distance from each other and from the branch for faster delivery time and save drivers roaming time on streets. Some people have conducted researches in this area. Boobalan, Lopeza, and Gao proposed to use structural and attribute similarity to identify the clusters in graph network [2].

\footnotetext{
* Corresponding author: Jiang Guo, jguo@calstatela.edu
} 
Bogarín, Romero, Cerezo, and Sánchez-Santillán proposed to use clustering to improve the educational models [3]. We use clustering/grouping algorithm to group orders to be delivered.

The SOOS offers important features to meet main objective, i.e. place and track orders, group orders, and track driver through mobile devices and web browsers. In order to accomplish this, the web interface of the system is divided into many categories of user roles:

- Support various types of customers to place and track orders, locate branches, get direction to desired destination, update profile and addresses.

- Support drivers to manage their delivery queue and find the routes of the delivery on the map.

- $\quad$ Support managers to manage orders and track drivers on map.

- Besides providing all manager privileges, support administrators and owner with special privileges to manage the entire system including functionalities such as manage users and items, changing roles and creating a new branch.

\section{SOOS System Architecture}

The SOOS is consisting of three applications: SOOS Web Application, SOOS-Air Android application and SOOS-Air-Gate Web Service (See Figure 1 and Figure 2).

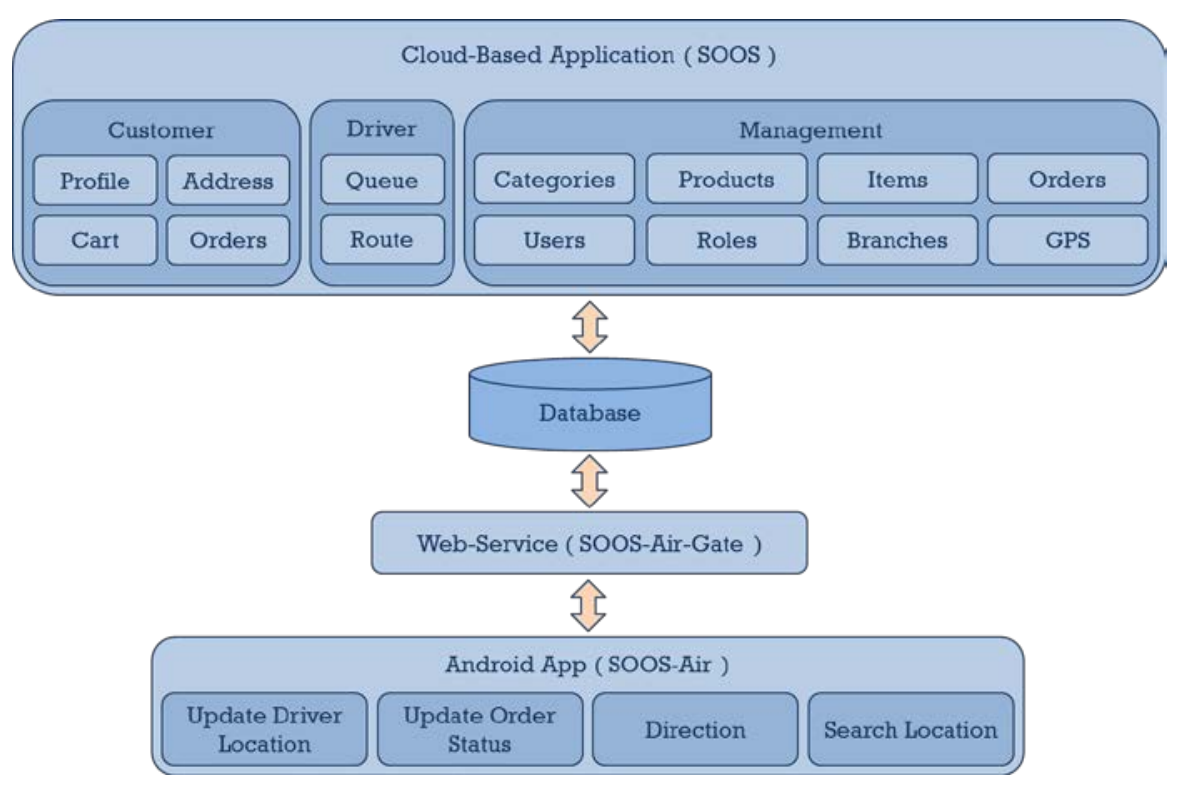

Figure 1: SOOS Components 


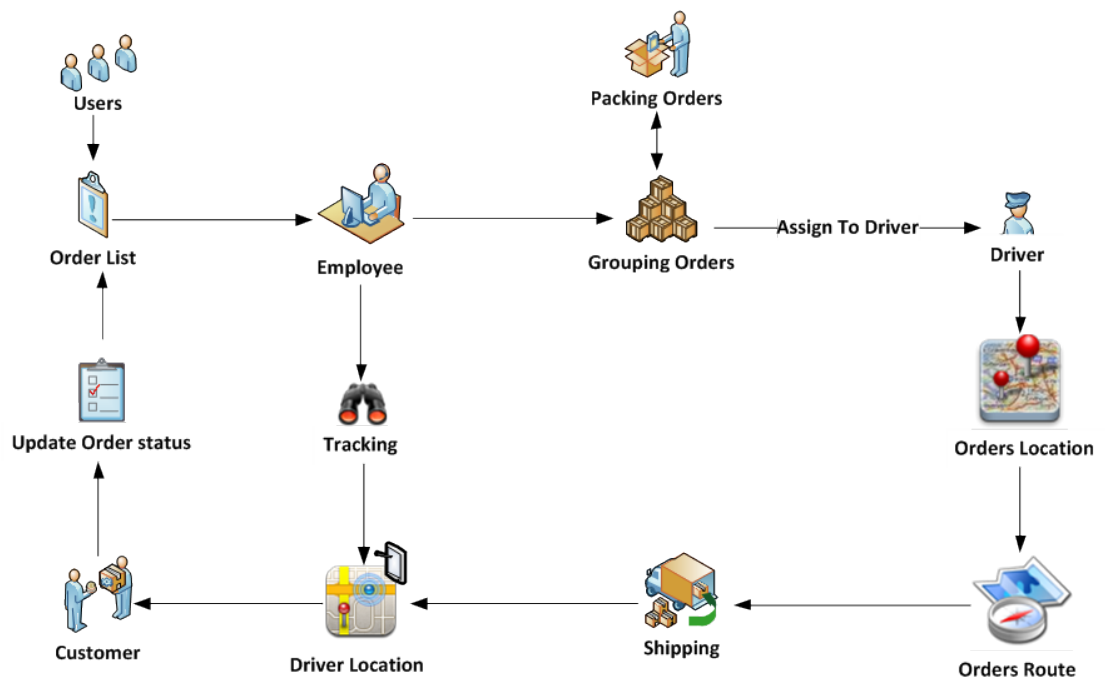

Figure 2: Flow Chart of Delivering Orders

\subsection{SOOS Web Application}

A user can access and order items in the Smart Order Online System through a Web-Browser from a desktop machine. The features are provided including:

- $\quad$ Search for item and add it to cart.

- $\quad$ Select the shipping address and the payment method.

- $\quad$ Place the order.

- $\quad$ Track the order.

The information about drivers locations including the statuses of every order are updated real-time using the data obtained from the SOOS-Air (Driver device). Right now, there are many companies providing tracking ability to customers, such as UPS, USPS, FedEx, and DHL. However, none of them provides real time and map tracking abilities. Our system uses GPS technology to provide this information.

\subsection{SOOS-Air Android App}

The mobile devices in driver hands update the current location frequently through the SOOS-Air App over TCP/IP connection, since TCP/IP is very reliable and efficient and used in cloud based applications, such as data centers [4]. The SOOS-Air sends the location information to the WebService (SOOS-Air-Gate), and then this service updates this information in the Smart Order Online System databases. Also, the drivers update the statuses of every order. The main application in the drivers' mobile devices is the Android App (SOOS-Air) that provides the drivers all the information of orders that he/she needs to deliver (See Figure 3).

The SOOS-Air App is an order status/tracking application that helps drivers keep updating their location and delivery statuses. It allows drivers to see the orders that are in the queue and their delivering dates. Drivers update the order information when the items have been delivered. GPS is used in SOOS-Air App, which is a widely used technology to implement tracking [5].

Drivers can use SOOS-Air to do the following: 
- View a list of orders assigned to them.

- Shows the order number, description, address and invoice amount.

- Show the order location on the map.

- Get the direction to the order address.

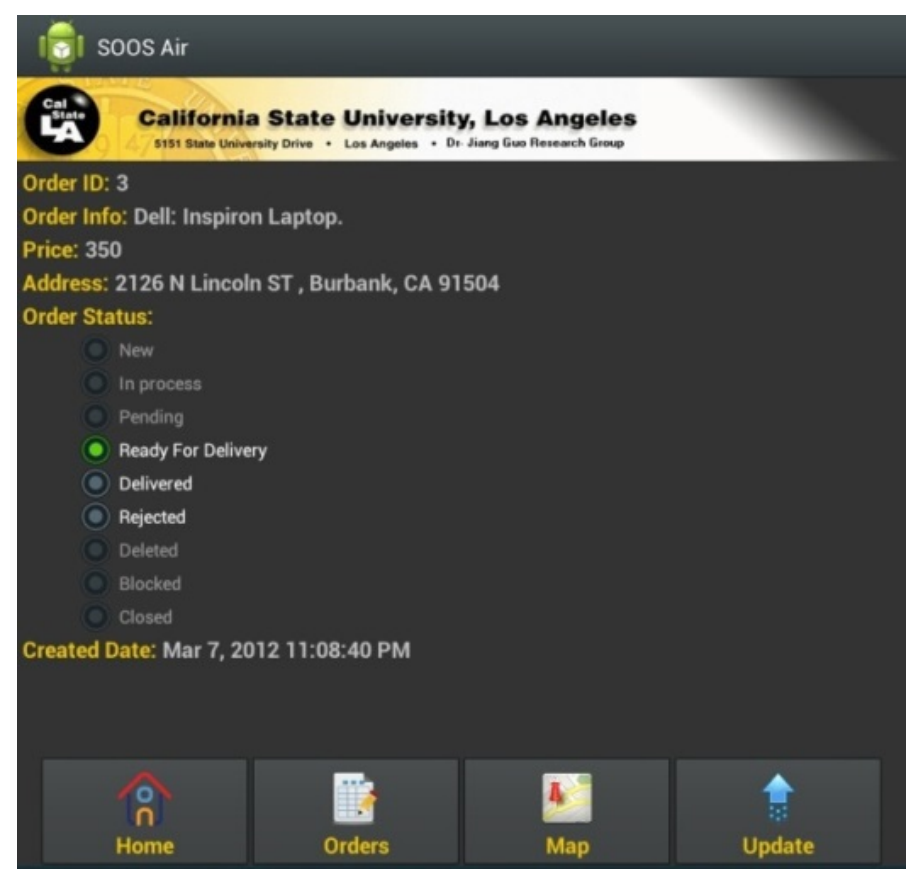

Figure 3: SOOS-Air App

\subsection{SOOS-Air-Gate Web Service}

The SOOS-Air-Gate Web Service is the mid-layer between the SOOS-Air and SOOS. SOOS-AirGate is designed as a mid-layer to support the communication between the SOOS-Air and SOOS Database over the Web.

It plays a role of bridge to update drivers' location and delivery information in real time. It is a bidirectional communication mechanism. One way, it retrieves order information from cloud based centralized data repository and sends them to the drivers. The other way, it gets the location and delivery information from drivers and updates the information in data repository.

\section{Integrated Google Map}

One of the main components of the system is the Google Map based subsystem that provides the customers, managers, and administrators a rich graphical representation of drivers' locations on a real time map. Customers can get the route and distance between branches and customers after they provide their locations. Google Map based subsystem supports more than just markers by providing an option to display an information window, which contains the information about the branches, drivers' locations upon clicking one of the markers. 
The customers can search and find information of items, find location and information of branches, get direction/routes between the branches after they provide their location information graphically on the map.

Figure 4 shows the marker where the branch located on the map. Upon clicking the marker, the customer can view the information about the branch on the left.

In our system, the drivers can arrange their deliveries route on the map system and use the GPS on mobile devices as guiders.

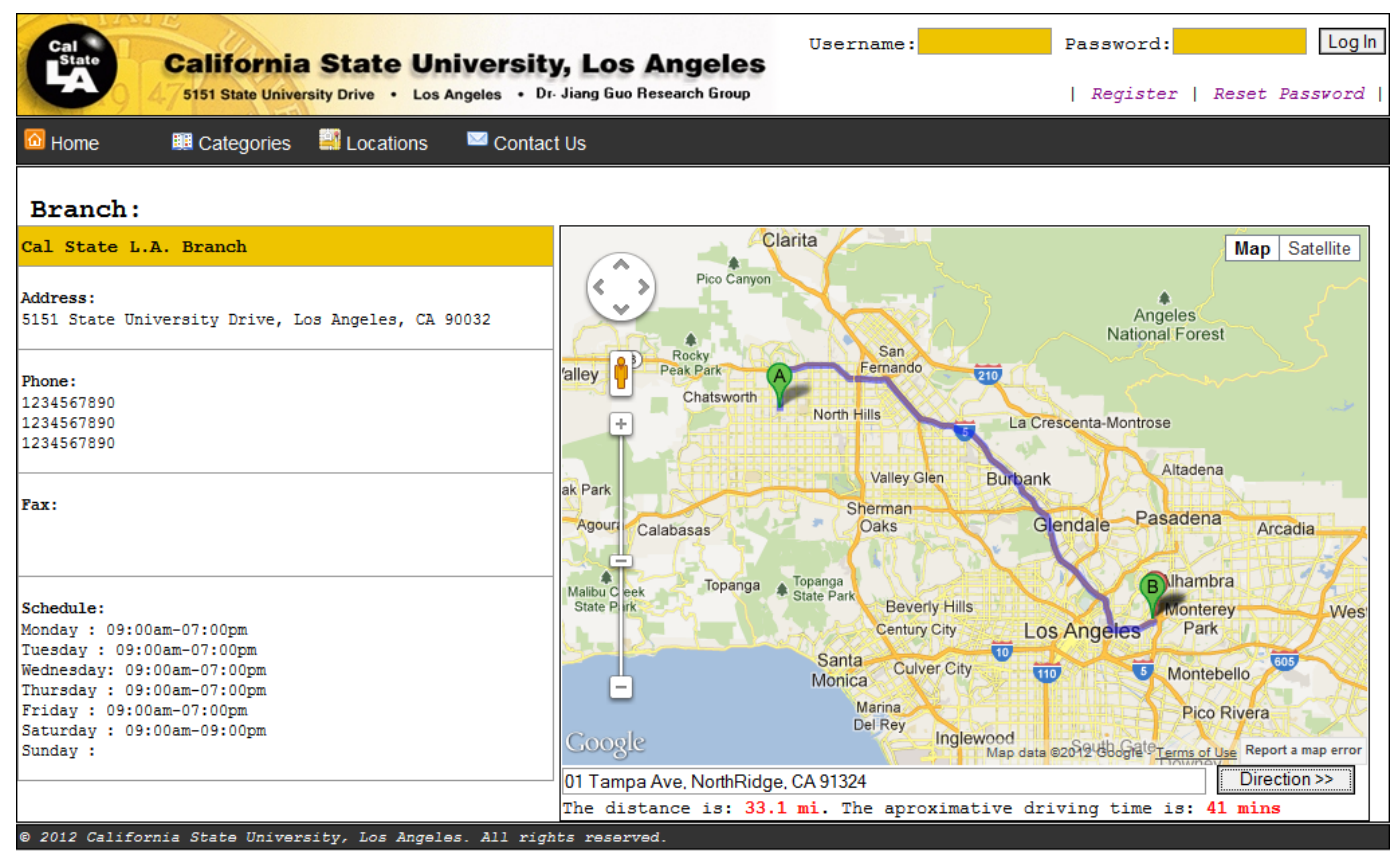

Figure 4: Google Map and GPS Based Tracking System

\section{Clustering/Grouping Algorithm}

Clustering is the most important task of assigning a set of addresses into groups (called clusters) so that the addresses in the same cluster are closer to each other than to those in other clusters. Clustering / Grouping algorithm could be applied when two or more orders need to be delivered by two or more drivers. Clustering addresses process allow deciding the group that the order should be belongs to. This algorithm will group orders based on their distance from each other for faster delivery time.

The following flowchart (See Figure 5) shows how arbitrary decisions taken by these algorithms during clustering. Assuming that there are $(\mathrm{N})$ addresses (Point), and we need to group them in $(\mathrm{G})$ group, then the algorithm will do the following steps.

Inputs:

$P=\left\{p_{1}, p_{2}, p_{3}, \ldots, p_{n}\right\}:$ Points to be clustered $\}, K:$ number of clusters, C: center

Procedure:

Set $G$ to initial value (set nearest $K$ point to $C$ as centroid). 
For each $p_{i} \in P\left(p_{i}\right.$ not in $\left.G\right)$

link $p_{i}$ to nearest group $G_{j}(j \in\{1 . . k\})$

If (size of $G_{j}=2$ )

End

set farthest point from $C$ as centroid

If (size of $G_{j}>2$ )

New Centroid $\geq \frac{\text { Nearest } p \text { from } C+\text { Farthest } p \text { from } C}{2}$

End

For each $p_{i} \in P\left(p_{i}\right.$ in $\left.G_{j}\right)$

End

re-link $p_{i}$ to nearest group

End

Outputs:

$G=\left\{G_{1}, G_{2}, G_{3}, \ldots, G_{k}\right\}:$ set of clusters/groups

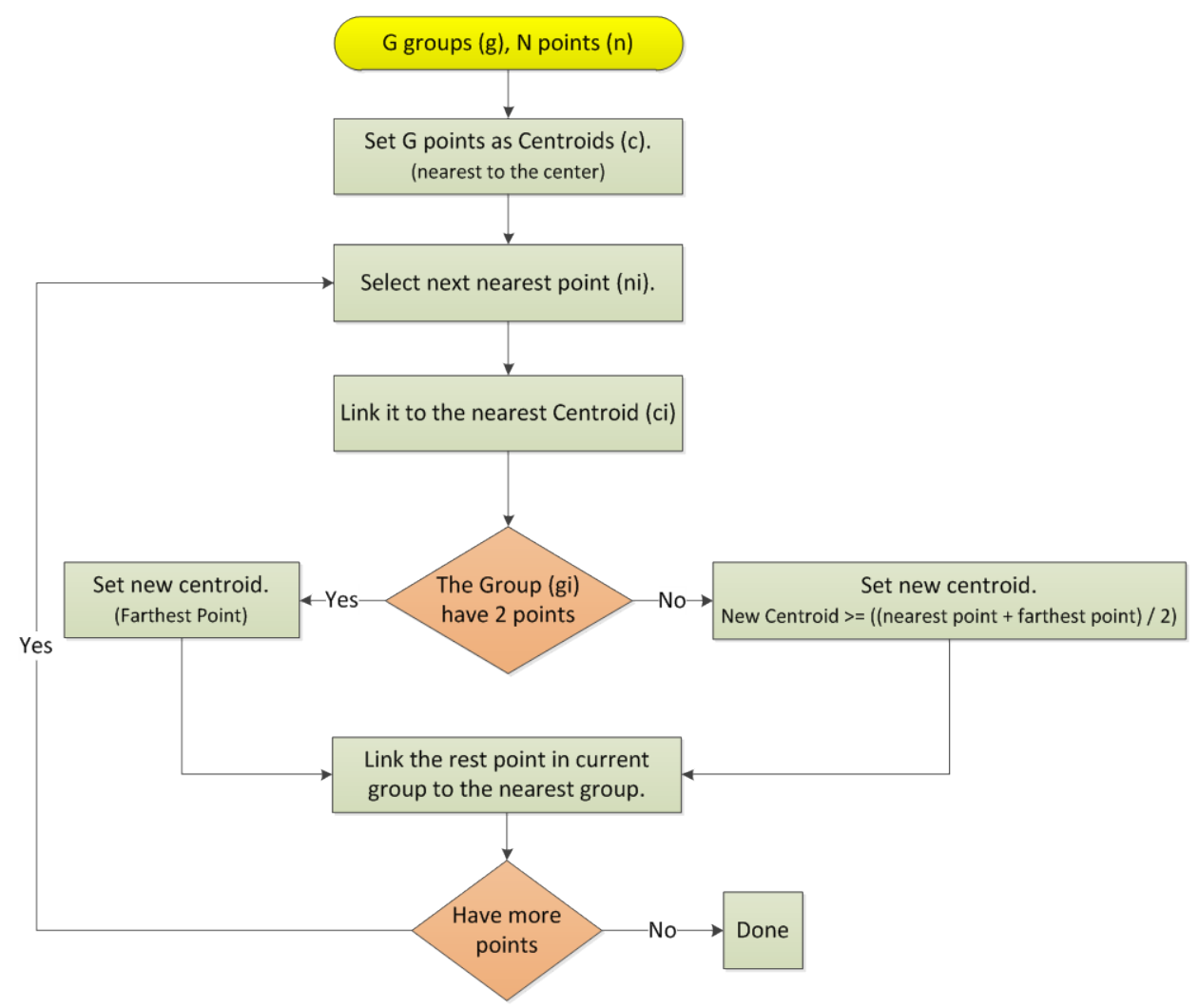

Figure 5: Clustering/Grouping Algorithm 


\section{Conclusion}

The Smart Order Online System is a prototype system to verify our creative ideas of map based clustering algorithm. It is powerful and scalable.

The SOOS is an advanced system. It is used as an efficient and user-friendly online system to implement our framework to verify the integration of map, GPS, Android app, and clustering abilities. The system is cloud based and open structured. It is flexible to add new features.

\section{Acknowledgements}

The work was supported in part by the NASA under grant NNX15AQ06A.

\section{References}

[1] William H. DeLone \& Ephraim R. McLean, "Measuring e-Commerce Success: Applying the DeLone \& McLean Information Systems Success Model", International Journal of Electronic Commerce, Volume 9, Issue 1, 2004.

[2] M. Parimala Boobalan, Daphne Lopeza, and X.Z. Gao, "Graph clustering using k-Neighbourhood Attribute Structural similarity", Applied Soft Computing, Volume 47, October 2016.

[3] Alejandro Bogarín, Cristóbal Romero, Rebeca Cerezo, Miguel Sánchez-Santillán, "Clustering for improving educational process mining", Proceedings of the Fourth International Conference on Learning Analytics and Knowledge, ACM New York, 2014.

[4] David Sidler, Zsolt István, Gustavo Alonso "Low-latency TCP/IP stack for data center applications", 26th International Conference on Field Programmable Logic and Applications (FPL), 2016.

[5] SeokJu Lee, Girma Tewolde, Jaerock Kwon, "Design and implementation of vehicle tracking system using GPS/GSM/GPRS technology and smartphone application", 2014 IEEE World Forum on Internet of Things (WF-IoT), 2014. 